\title{
Anabases
}

ANABASES Traditions et réceptions de l'Antiquité

$9 \mid 2009$

Varia

\section{Les allusions et citations latines dans Les Aventures d'Astérix le Gaulois}

Benoît Jeanjean

\section{(2) OpenEdition}

Journals

Édition électronique

URL : http://journals.openedition.org/anabases/537

DOI : 10.4000/anabases.537

ISSN : 2256-9421

Éditeur

E.R.A.S.M.E.

\section{Édition imprimée}

Date de publication : 1 mars 2009

Pagination : 296-302

ISSN : 1774-4296

\section{Référence électronique}

Benoît Jeanjean, "Les allusions et citations latines dans Les Aventures d'Astérix le Gaulois ", Anabases [En ligne], 9 | 2009, mis en ligne le 01 mars 2012, consulté le 20 octobre 2019. URL : http:// journals.openedition.org/anabases/537 ; DOI : 10.4000/anabases.537

Ce document a été généré automatiquement le 20 octobre 2019.

(c) Anabases 


\title{
Les allusions et citations latines dans Les Aventures d'Astérix le Gaulois
}

\author{
Benoît Jeanjean
}

1 Le succès des Aventures d'Astérix le Gaulois, le personnage créé par René Goscinny et Albert Uderzo, a dépassé depuis longtemps les limites de la Gaule. Traduit dans une multitude de langues, notre Gaulois national ne s'est pas contenté de « résister encore et toujours » aux légions de Jules César, il a encore poussé l'audace jusqu'à conquérir le monde. Les raisons de ce succès sont nombreuses, depuis la qualité du trait d'Uderzo, tout en souplesse et en clarté, jusqu'à la finesse des dialogues de Goscinny, qui a su habilement mêler à la conduite du récit de multiples jeux de mots et allusions aux mondes antique et contemporain. Or, parmi les références à l'Antiquité qui émaillent la série, on rencontre très souvent des citations latines qui contribuent tout particulièrement à renforcer l'effet « Rome antique ». Pourrait-on, en effet, imaginer une BD où les Romains ne parleraient pas latin, ne serait-ce qu'occasionnellement ${ }^{1}$ ? Plutôt qu'à un relevé exhaustif des citations et des allusions à l'histoire et à la littérature latine $e^{2}$, c'est à un parcours à travers quelques exemples significatifs que je convie le lecteur. On y verra que la part faite à Jules César, à la fois personnage historique et écrivain, est considérable et que si les auteurs latins, poètes et prosateurs, sont largement sollicités, les citations bibliques ne manquent pas non plus. La question de savoir si Goscinny connaissait le latin importe peu ici, et il serait vain de tenter de prouver quoi que ce soit à partir de citations qui proviennent toutes, ou presque, des fameuses « pages roses » du dictionnaire. Il est en revanche très intéressant d'observer comment ces citations sont exploitées dans les albums d'Astérix, car, loin d'y avoir un rôle de pur ornement, elles jouent souvent sur l'intertextualité pour donner une dimension supplémentaire à l'humour déployé dans la série. 


\section{César, auteur de La Guerre des Gaules et... de quelques mots historiques}

2 La figure de César est centrale dans Astérix et il est inutile d'énumérer tous les albums où elle apparaît. Le personnage est, bien sûr, présenté avant tout comme le vainqueur de Vercingétorix à Alésia, mais il ne faut pas oublier que la fameuse scène de la reddition du chef gaulois qui jette ses armes aux pieds du glorieux général romain ${ }^{3}$ est directement issue de l'œuvre que César rédigea lui-même pour présenter ses huit années de campagnes militaires en Gaule : La Guerre des Gaules. Or, il se trouve qu'un certain nombre de vignettes font directement référence à cet ouvrage. C'est notamment le cas dans Le Bouclier arverne, lorsque César - le personnage cette fois cherche à rappeler aux Gaulois sa victoire de 52 avant J.-C. en paradant, debout sur le bouclier de Vercingétorix. Le seul problème est qu'il ne sait pas où se trouve ce bouclier. Il envoie donc quelqu'un chercher pour lui dans la salle où il entrepose le butin de ses campagnes, mais celui-ci revient bredouille, comme nous pouvons le constater dans la vignette suivante (p. 18, vignette 8) :

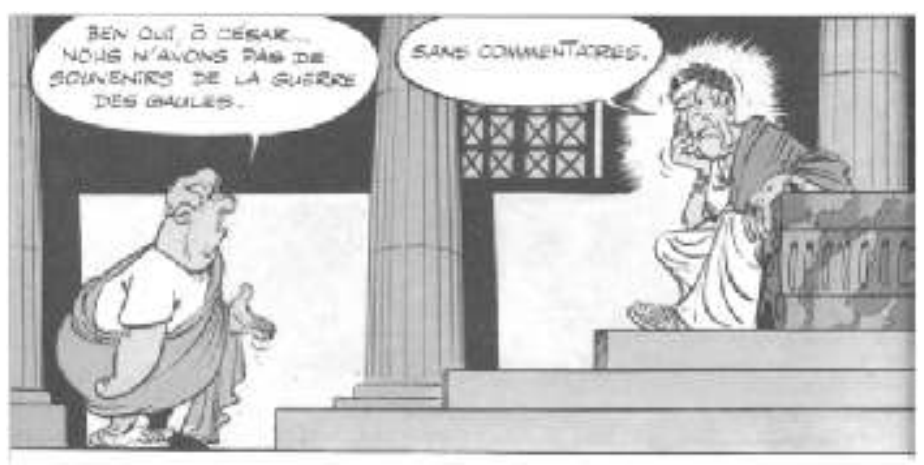

3 L'allusion à la guerre des Gaules est transparente, mais, dans la bouche du tribun Fanfrelus, il s'agit davantage de l'événement historique que du récit écrit par César. La réponse désabusée de ce dernier prend cependant une tout autre dimension, dès lors que l'on sait que le titre complet de son ouvrage n'est pas La Guerre des Gaules, mais les Commentaires sur la guerre des Gaules. D'un seul coup, c'est comme si César oubliait qu'il avait lui-même écrit un livre sur la question!

Cet oubli, plein d'amère ironie, est sans doute bien provisoire, puisque dans Le Domaine des dieux il retrouve son statut d'auteur et se propose, dès la première vignette de la première page (p.5), de «faire quelques petits commentaires » à ses conseillers sur la situation en Gaule. C'est d'ailleurs à cette occasion qu'on rencontre une autre allusion à La Guerre des Gaules dans un petit dialogue sibyllin dont le sens échappe aux lecteurs peu avertis (p. 5, vignettes 3 et 4 ) : 

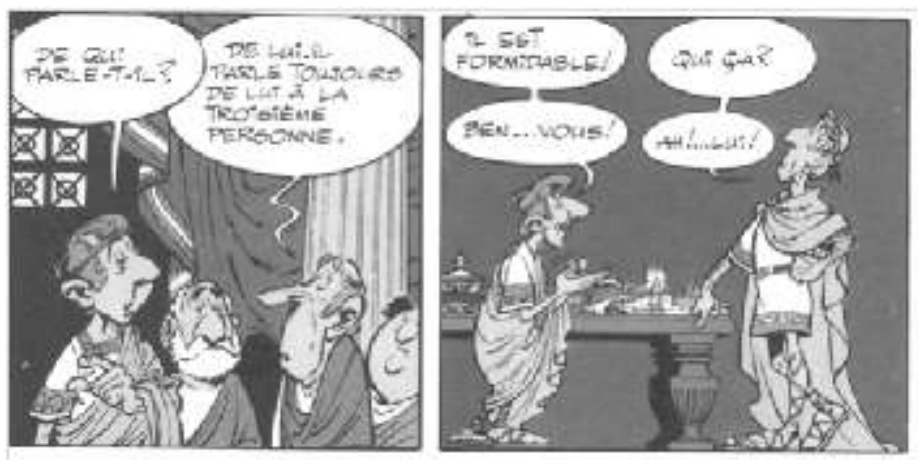

César vient en effet de rappeler, à la troisième personne du singulier, ses propres exploits militaires en Gaule sans se désigner nommément. Cela explique la question de son plus jeune conseiller qui, visiblement, n'a pas lu ses Commentaires sur la guerre des Gaules. S'il avait lu l'ouvrage, il saurait que César n'y parle jamais à la première personne, mais toujours à la troisième, ce qui contribue à donner une allure objective à son récit pourtant très partial !

On rencontre une dernière allusion aux Commentaires sur la guerre des Gaules dans le dernier Astérix réalisé par le duo Goscinny-Uderzo : Astérix chez les Belges. On se souvient des premières pages de l'album où l'on apprend que les légions de César sont parties combattre les Belges et que les légionnaires qui reviennent en Armorique s'y sentent au repos, loin des combats acharnés qu'ils ont connus sur le front de l'Est. Cela agace au plus haut point Abraracourcix, qui s'emporte, et Bonnemine tente de le calmer en lui rappelant en quelle estime César les tient, lui et ses hommes. Malheureusement, Astérix a entendu dire que César est d'un autre avis, qu'il rapporte immédiatement (p.9, vignette 8) :

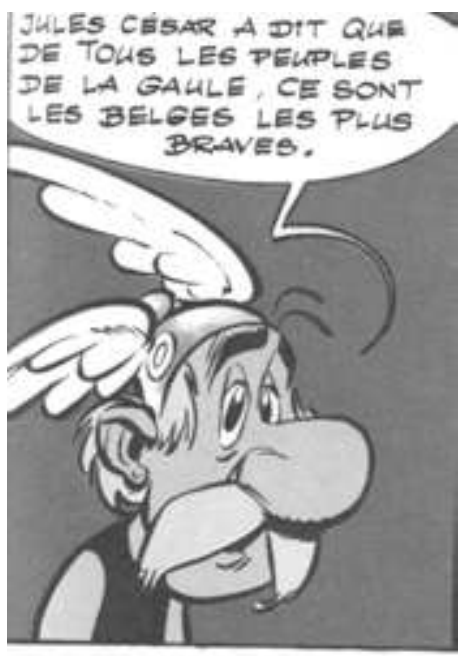

7 On pourrait penser que César n'a jamais rien dit de tel et qu'il ne s'agit que d'un prétexte pour envoyer les héros en Belgique, mais il se trouve que l'affirmation de la bravoure des Belges figure bel et bien au livre 1 des Commentaires sur la guerre des Gaules. Le premier chapitre de ce livre expose l'organisation territoriale de la Gaule dont l'espace est habité par trois peuples distincts : les Belges, les Aquitains et les Gaulois ou Celtes. César ajoute alors: «Les plus braves [fortissimi] de ces trois peuples sont les Belges, parce qu'ils sont les plus éloignés de la province romaine et des raffinements de sa civilisation, que les marchands y vont très rarement et, par conséquent, n'y introduisent pas ce qui est propre à amollir les cœurs, et qu'ils sont les plus voisins des 
Germains, qui habitent sur l'autre rive du Rhin et avec qui ils sont continuellement en guerre» (Guerre des Gaules I, 1). Cette affirmation sert donc de point de départ à l'intrigue d'Astérix chez les Belges. Mais que les lecteurs se rassurent, les propos de César ne font qu'établir une hiérarchie de la bravoure en Gaule, ils ne mettent aucunement en cause celle des Gaulois !

En plus de ses exploits militaires, César est également célèbre pour quelques mots historiques qui se transmettent dans les classes de latin de nos collèges. Le plus fameux est sans doute celui qui aide les élèves à retenir les formes du parfait des verbes latins uenio, uideo et uinco (venir, voir et vaincre) et que l'on trouve, par exemple, dans Astérix en Hispanie (p. 6, vignette 3 ) :

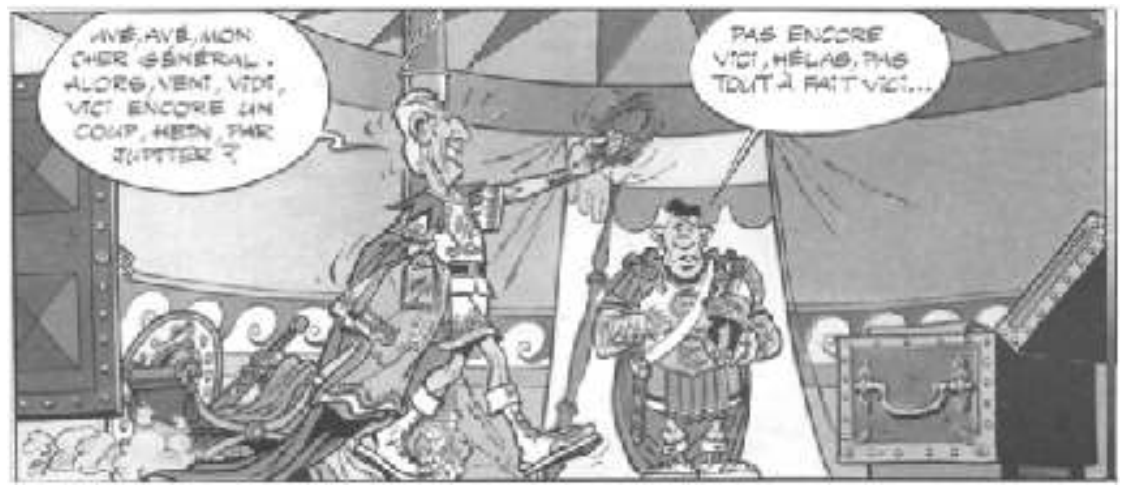

9 Si la formule est synonyme de victoire totale et définitive, on ignore souvent qu'elle servit d'abord à désigner la rapidité avec laquelle César vint à bout de Pharnace, roi du Pont et fils de Mithridate, comme en témoigne l'historien Suétone dans ses Vies des douze Césars: "Pour son triomphe ${ }^{4}$ du Pont, entre autres objets présentés dans le cortège, il fit porter devant lui une inscription avec ces trois mots : Veni, uidi, uici [Je suis venu, j'ai vu, j'ai vaincu], soulignant ainsi la rapidité de cette campagne au lieu d'en énumérer les faits comme pour les autres » (Vie de César, 37). Dans Astérix, on constate que César ne peut jamais reprendre la formule jusqu'au bout, car inévitablement il y a un petit village " qui résiste encore et toujours » et qui lui interdit de triompher !

On prête également à César un second mot fameux qu'on retrouve dans Astérix et Cléopâtre, dans la bouche de Triple-Patte, pirate et latiniste distingué à ses heures (p. 10, vignette 7) :

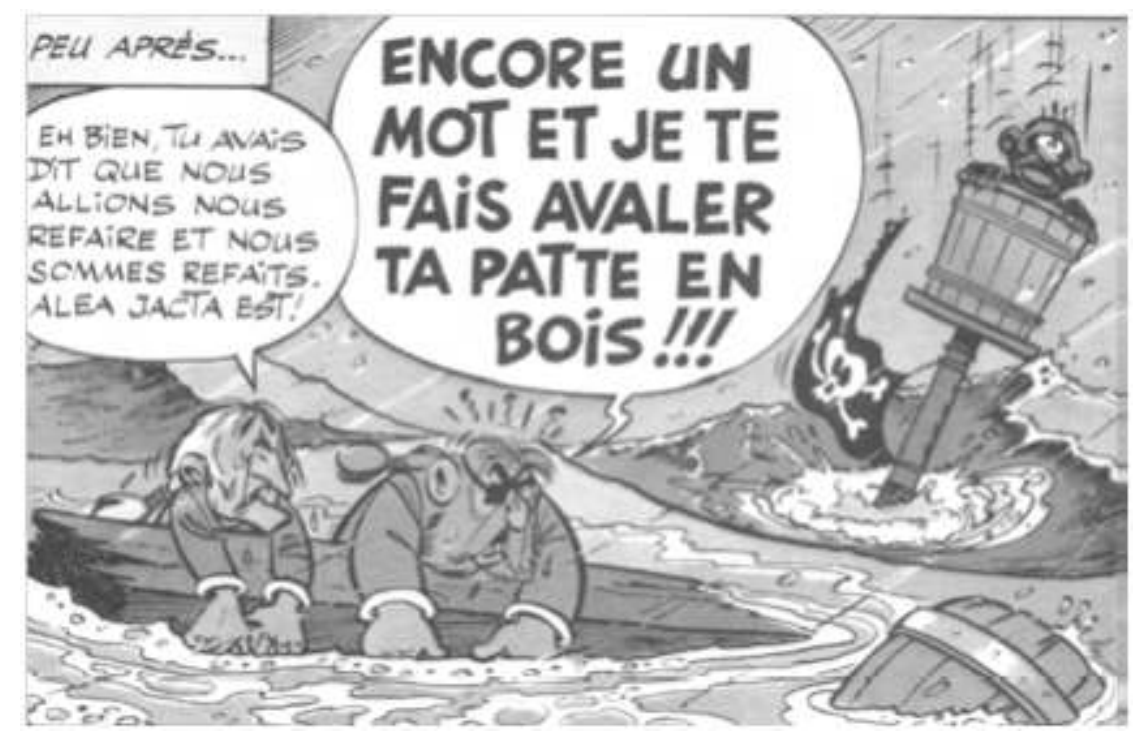


11 situation contre laquelle il n'y a plus rien à faire. Ce fatalisme ne correspond pas au sens beaucoup plus audacieux et positif que César donne à la formule lorsqu'il l'utilise, en 49 avant J.-C., au moment où il décide de franchir le Rubicon et d'engager, du même coup, les hostilités avec Pompée. C'est encore une fois l'historien Suétone qui rapporte la scène : "Comme il hésitait, il reçut un signe d'en haut. Un homme d'une taille et d'une beauté extraordinaires apparut soudain [...]; cet homme prit à l'un d'entre eux son instrument, s'élança vers la rivière et, sonnant la marche avec une puissance formidable, passa sur l'autre rive. Alors César dit : "Allons où nous appellent les signes des dieux et l'injustice de nos ennemis. Alea jacta est [Le sort en est jeté]". » (Vie de César, 32). On le voit bien, César est loin d'être fataliste lorsqu'il lance ces mots qui écartent toute idée de hasard. Il se montre au contraire providentialiste, puisqu'il s'engage dans la direction que lui indiquent les dieux.

12 systématiquement, dans Astérix, lorsqu'il se trouve en présence de Brutus et qu'on rencontre en latin dans Astérix gladiateur (p. 38, vignettes 4 et 5) :
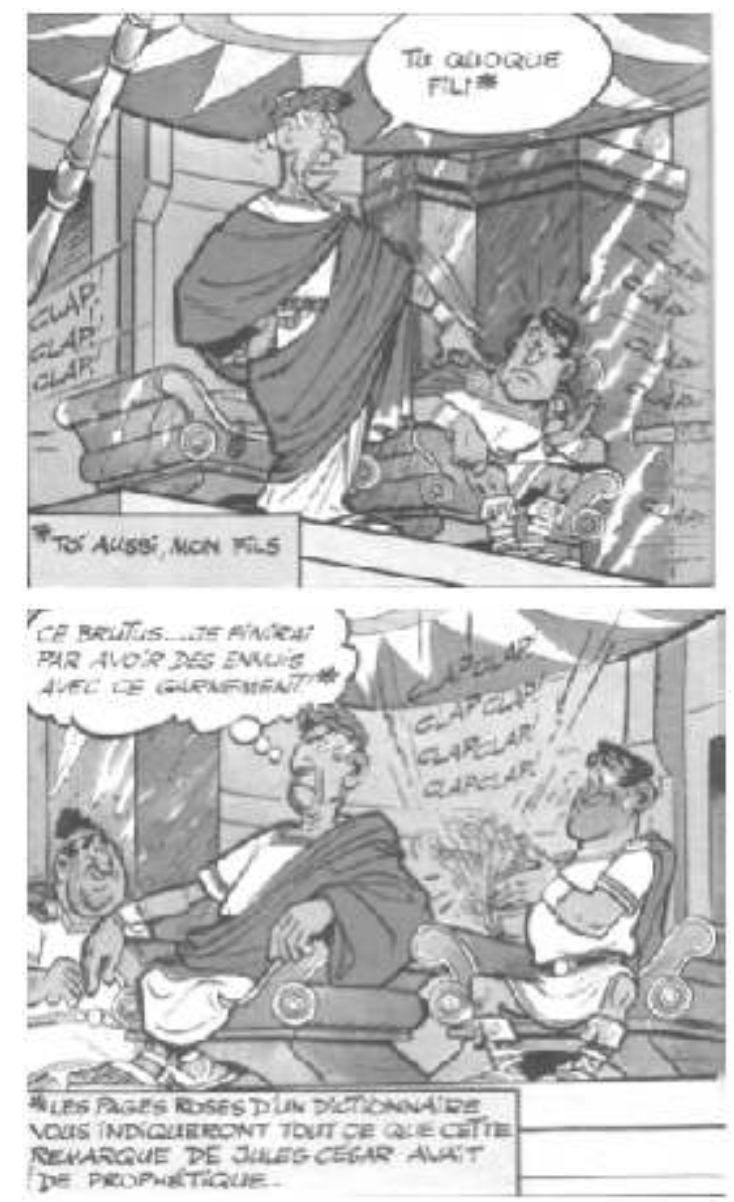

La formule revient à nouveau dans une page de La Zizanie (p. 6, vignettes 4 et 5) : 

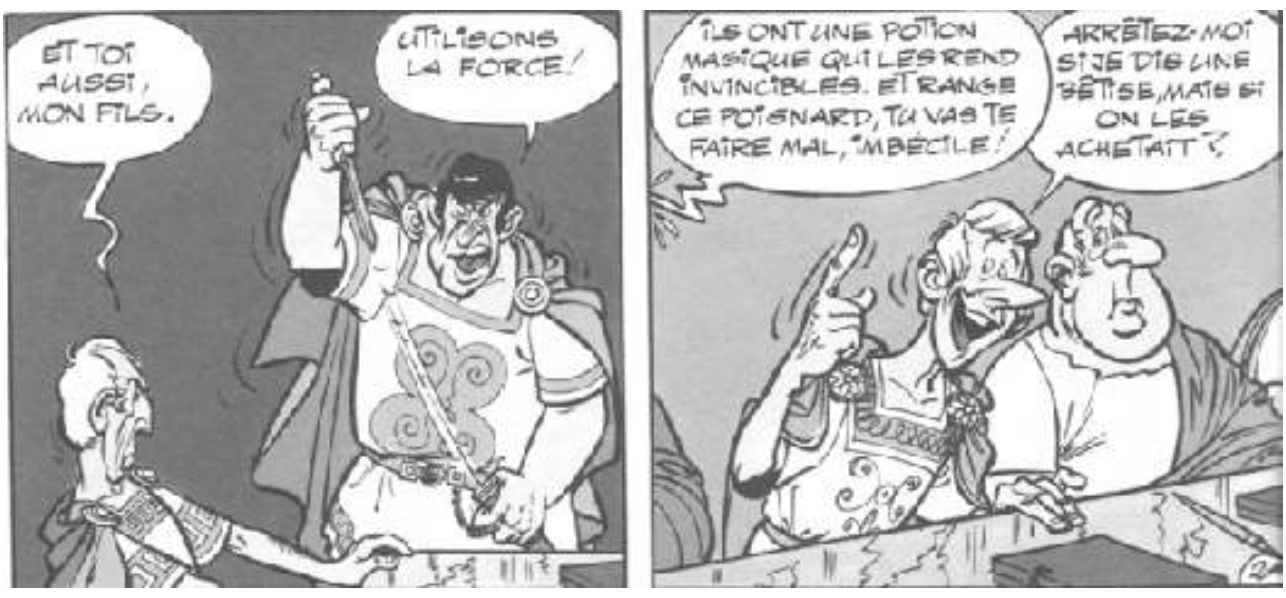

La présence de Brutus, fils adoptif de César, est le déclencheur de la formule derrière laquelle Goscinny ne cache pas, sur le ton de l'humour, qu'une menace se profile. Les "pages roses" d'un dictionnaire n'indiquent plus nécessairement en quoi consiste cette dernière, mais elle se précise immédiatement lorsqu'on connaît le passage où Suétone rapporte la mort de César, qui fut assassiné par une conjuration de sénateurs au nombre desquels se trouvait précisément Brutus : «Il fut ainsi percé de vingt-trois blessures, n'ayant poussé qu'un gémissement au premier coup, sans une parole; pourtant, d'après certains, il aurait dit à Marcus Brutus qui se précipitait sur lui : "Toi aussi, mon fils !" "(Vie de César, 82).

Le paradoxe de cette citation latine, c'est que, à en croire Suétone, elle n'aurait pas été prononcée en latin, mais en grec, car César maîtrisait parfaitement les deux langues. Il aurait donc dit Kai su, teknon!, et non Tu quoque fili !

\section{Le mot du poète}

Les «mots de César» nous sont rapportés par l'historien Suétone, et nombre de citations latines d'Astérix sont tirées d'autres prosateurs parmi lesquels Cicéron n'est pas le moins célèbre. Cependant, les « pages roses » du dictionnaire, si elles sont bien la source de Goscinny, fournissent un grand nombre de citations de poètes qui fleurissent tout au long des différents albums. Je n'en donnerai qu'un exemple tiré du plus célèbre poète de l'époque de l'empereur Auguste.

Il se rencontre dans Astérix légionnaire, où l'un de ses vers est systématiquement associé par les Romains au nom de Tragicomix, le fiancé de Falbala, enrôlé de force dans la légion pour aller combattre loin de chez lui, en Afrique. Astérix, qui cherche à savoir où il a été envoyé, s'informe auprès du bureau des renseignements de la légion, à Condate (p. 17, vignette 2) : 


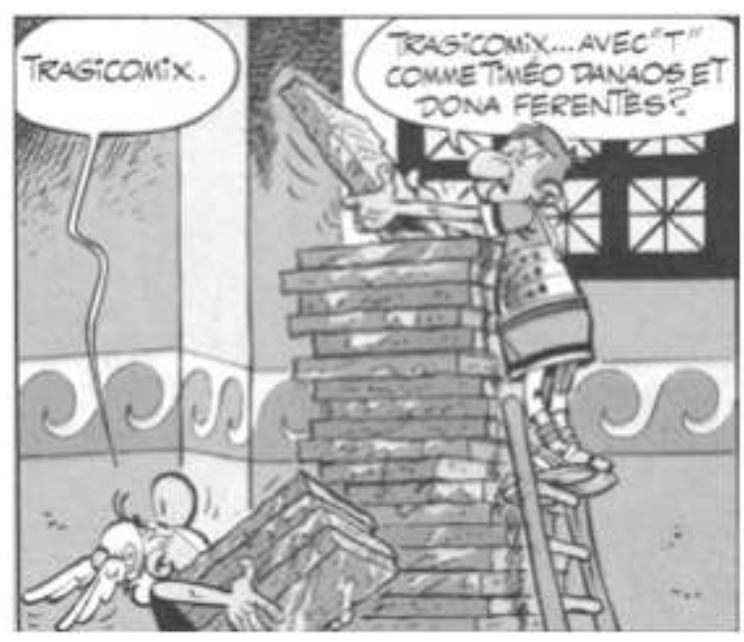

La formule Timeo Danaos et dona ferentes (Je crains les Grecs, même s'ils apportent des cadeaux) est de Virgile, et elle est bien connue des latinistes, car elle sert d'exemple type depuis des générations pour illustrer la valeur adverbiale de la conjonction et. Elle fonctionne donc comme un automatisme dès que la question du et adverbial reparaît. De la même façon, dans Astérix légionnaire, la citation revient lorsque le nom de Tragicomix est prononcé. Virgile, toutefois, ne l'a pas formulée pour servir d'exemple à un fait de la langue latine, mais bien pour servir la cause d'un récit épique où l'on assiste, avec le fameux épisode du cheval de Troie, à la prise de cette ville par les Grecs. Énée, qui le raconte à Didon, arrive au moment où les Grecs, feignant d'avoir perdu tout espoir de victoire, ont quitté les abords de Troie en laissant un cheval de bois devant la porte de la ville. Les Troyens, voyant dans ce cadeau un signe d'hommage des Grecs aux divinités qui protègent leurs ennemis, décident de le faire entrer dans la cité. Mais un Troyen, Laocoon, soupçonne une ruse et en avertit ses concitoyens en ces termes: "Vous croyez les ennemis partis? Ou pensez-vous que les offrandes des Danaens soient jamais exemptes d'artifices? Est-ce ainsi que vous connaissez Ulysse ? [...] Ne vous fiez pas à ce cheval, Troyens. Quoi qu'il en soit, je crains les Danaens même quand ils portent des offrandes" (Énéide 2, 43-49). L'avertissement de Laocoon ne sauva malheureusement pas les Troyens qui refusèrent de croire que le cheval pouvait présenter une menace pour leur ville. On est bien loin, on le voit, du réflexe mécanique qui associe systématiquement un nom ou un fait de langue à une tournure. Mais si le Romain d'Astérix légionnaire est loin de Virgile, son réflexe rejoint en revanche celui du latiniste d'hier et d'aujourd'hui.

\section{Un mot de la Bible}

À côté des citations des poètes, les "pages roses " font la part belle aux citations bibliques et celles-ci sont nombreuses dans les pages d'Astérix. J'en signalerai une qui illustre l'Ancien Testament. Dans Astérix gladiateur, tout d'abord, Astérix et Obélix ont pris place à bord d'un navire marchand phénicien qui les conduit vers Rome. Or, pendant la traversée, ils sont attaqués par des pirates convaincus d'avoir affaire à une proie facile et qui se permettent de vendre la peau de l'ours avant de l'avoir tué (p. 15, vignette 8$)$ :

Image 10.wmf 
20 La citation de Triple-Patte - célébrissime dans la peinture qui en a fait, avec les "vanités ", un thème de prédilection - se trouve dans les tout premiers versets du livre de Qohéleth (autrement appelé l'Ecclésiaste), livre qui souligne, sous forme de nombreuses maximes, la fragilité et le caractère éphémère de la vie humaine. L'ensemble du livre est placé sous le signe du verset qui l'ouvre: "Vanité des vanités dit Quohélet, tout est vanité » (Qo. 1, 2). Placée dans la bouche d'un pirate, la citation prend sa pleine dimension : l'abordage du navire marchand doit rendre vains les rêves de fortune nourris par les marchands phéniciens, qui pensaient voguer vers de juteuses affaires et vont se retrouver eux-mêmes réduits au rang de marchandises, s'ils survivent à l'affaire. Mais la vanité est ici à double face. Triple-Patte ignore que la citation va se retourner contre lui (et pour cause : c'est la première fois que les pirates rencontrent les Gaulois !), car s'il ironise sur la vanité des marchands phéniciens, c'est de lui qu'il parle sans le savoir, puisque les espoirs de butin facile qu'il nourrit avec ses compagnons vont se révéler vains à leur tour.

N'est-ce pas là l'illustration d'un nouveau proverbe qui s'impose ici et qui nous fournira le mot de la fin : "Vanité bien ordonnée commence par soi-même ! »

\section{NOTES}

1. Il est vrai qu'au régime de baffes auquel ils sont soumis, ils « en perdent leur latin » (cf. Astérix le Gaulois, p. 5, vignette 10).

2. Un tel relevé a été amorcé dans le Livre d'or d'Astérix, sur une idée d'O. ANDRIEU, Éditions AlbertRené, Paris, 1999 ; mais il n'est pas exhaustif, comporte quelques erreurs et surtout, n'indique jamais le texte antique d'où sont tirées les citations.

3. Scène qui fait l'objet d'un traitement variable selon les albums où elle se présente. Ainsi, César n'est pas à son avantage dans Astérix le Gaulois (p.5, vignette 2), où il s'en faut de peu que les armes de Vercingétorix ne lui écrasent les pieds. La scène se répète, presque à l'identique, dans Le Bouclier arverne (p. 5, vignette 1), mais cette fois, César n'a pas le temps de retirer ses pieds ! Il faut dire que, dans les deux cas, le narrateur adopte le point de vue chauvin du descendant des Gaulois qui n'a toujours pas digéré la défaite de Vercingétorix. Une troisième occurrence de la scène tourne toutefois à l'avantage de César, dans Le Domaine des dieux (p. 5, vignette 2); mais il n'y a rien d'étonnant à cela, puisque cette fois, c'est César lui-même qui se charge d'en faire le récit!

4. Rappelons que, dans la Rome antique, le triomphe est la cérémonie au cours de laquelle un général victorieux est autorisé, par le Sénat, à célébrer publiquement sa victoire. Cette cérémonie est l'occasion de présenter au peuple le butin remporté et les prisonniers capturés lors de la campagne. 


\section{AUTEUR}

\section{BENOÎT JEANJEAN}

Université de Bretagne occidentale (Brest)

benoit-jeanjean@univ-brest.fr 